\title{
Methadone maintenance treatment program in prisons from the perspective of medical and non-medical prison staff: a qualitative study in Iran
}

\author{
Ghobad Moradi ${ }^{1}$, Marzieh Farnia ${ }^{2}$, Mostafa Shokoohi $^{3}$, Mohammad Shahbazi $^{4}$, Babak Moazen $^{5,6^{*}}$, \\ Khaled Rahmani ${ }^{7}$
}

\begin{abstract}
Background: As one of the most important components of harm reduction strategy for high-risk groups, following the HIV epidemics, Methadone Maintenance Treatment (MMT) has been initiated in prisoners since 2003. In this paper, we aimed to assess the advantages and shortcomings of the MMT program from the perspective of people who were involved with the delivery of prison healthcare in Iran.

Methods: On the basis of grounded theory and through conducting 14 Focus Group Discussions (FGDs), 7 FGDs among physicians, consultants, experts, and 7 FGDs among directors and managers of prisons $(\mathrm{n}=140)$ have been performed. The respondents were asked about positive and negative elements of the MMT program in Iranian prisons.

Results: This study included a total of 48 themes, of which 22 themes were related to advantages and the other 26 were about shortcomings of MMT programs in the prisons. According to participants' views "reduction of illegal drug use and high-risk injection", "reduction of potentially high-risk behaviors" and "making positive attitudes" were the main advantages of MMT in prisons, while issues such as "inaccurate implementation", "lack of skilled manpower" and "poor care after release from prison" were among the main shortcomings of MMT program.

Conclusions: MMT program in Iran's prisons has achieved remarkable success in the field of harm reduction, but to obtain much more significant results, its shortcomings and weaknesses must be also taken into account by policy-makers.

Keywords: Methadone Maintenance Treatment (MMT), Harm Reduction, Prisoners, Substance Use, High-Risk Behaviors, HIV

Copyright: (C) 2015 by Kerman University of Medical Sciences

Citation: Moradi G, Shokoohi M, Farnia M, Moazen B, Shahbazi M, Rahmani K. Methadone maintenance treatment program in prisons from the perspective of medical and non-medical prison staff: a qualitative study in Iran. Int J Health Policy Manag. 2015;4(9):583-589. doi:10.15171/ijhpm.2015.60
\end{abstract}

Article History:

Received: 24 November 2014

Accepted: 7 March 2015

ePublished: 12 March 2015

\section{Key Messages}

Implications for policy makers

- Because of the mentioned positive feedback from the prisons' staff, it is recommended that prisons continue the implementation of Methadone Maintenance Treatment (MMT) program.

- It is found that healthcare providers and managers of the prisons agreed with the MMT program, but the main concerns go to the time after releasing from prison which was considered as one of the main drawbacks of this program form the participants' view.

- There is a need for skilled staff, using specific guidelines with certain objectives and exact implementation plans to achieve more success.

\section{Implications for public}

Those people who were responsible for delivering of prison healthcare believed that Methadone Maintenance Treatment (MMT) programs, as a form of drug replacement therapy for opioid dependent individuals inside and outside prisoners, had remarkable benefits in reducing drug-related harms, risky behaviors and practices, lowering criminal behaviors, controlling HIV transmission and improving the lifestyle of the prisoners and their family. Thus, families' support and continuity of care and treatment can improve the effectiveness of this program before and after release from prison. Therefore, despite all necessary educational programs for the patient/prisoners, all family members also need to be educated about the advantages of the program.

\section{Background}

Prisoners are considered as one of the high-risk groups who are prone to the acquisition and further transmission of HIV and other infectious diseases in many countries $(1,2)$. Increased prevalence of HIV in prisoners, in comparison with general population, has been reported in the USA (ratio 6:1), and France (ratio 10:1) (3). In Iran, the prevalence of HIV has been reported as $1.28 \%(1)$ and $2.8 \%$ (4) in separate studies. Prisoners, especially those who inject drugs, are potentially at higher-risk of HIV infection (5-7). HIV outbreaks among 
People Who Inject Drugs (PWIDs) in prisons have been reported in Iran (8) and other countries $(9,10)$. Given the status of HIV epidemic among high-risk groups, especially prisoners, Iranian health authorities have tried to advocate for exhaustive control and prevention measures in- and out-side prisons. Since the early years of 2000s, policy-makers in Iran have established several harm reduction programs, among which Methadone Maintenance Treatment (MMT) has been one of the leading and important programs (11).

Nowadays, MMT is used in different parts of the world to reduce harm among PWIDs inside and outside prisons. MMT is one of the most important measures of harm reduction programs, and has led to effectively reduction or termination of drug injection and shared practices (12-14) and also highrisk sexual behaviors (12,15-19). This medication improves mental and physical conditions of patients and reduces the risk of diseases such as HIV/AIDS and hepatitis (20,21). According to studies, this method of treatment decreased mortality resulted from overdose, prevented withdrawal syndrome $(22,23)$ and re-incarceration $(24,25)$, and inhibited crimes initiated by illegal drug abuse; as a result, it provided infected people with the chance to improve their social health and life and involve in productive activities (26-30).

Since early 2000s, multiple harm reduction programs including Needle and Syringe Exchange Program (NSEP), MMT, referral system to Voluntary Counseling and Testing centers (VCTs), psychological and mental counseling, and some other services like condom distribution have been designed to reduce HIV transmission from high-risk groups like PWIDs and prisoners to their partners and other potentially at risk individuals both within and outside prison $(11,31)$. Throughout the Middle East and North Africa (MENA) region, harm reduction programs in Iran have been regarded as paradigms of best practices with remarkable impact in decelerating the propagation of HIV infection among PWIDs in- and out-side prisons (32). By the end of 2012, under the terms of Needle and Syringes exchange Program (NSP), more than 550 service centers had been distributing free needle and syringes among high-risk people in the community. However, based on the bio-behavioral surveys in Iran prisons in 2009 and 2013, inmates selfreportedly acknowledged unsafe and shared injection in- and out-side prison. Given the implementation of harm reduction programs in Iran, MMT's coverage among those who used or injected drugs grew up steadily from 2001 through the end of 2012, so that around half a million drug users had received free MMT services (in- and out-side prisons) through more than 4,000 centers (33); however, injecting drug use is still the main mode of HIV transmission in Iran (accounted for more than $60 \%$ of new HIV infections), which accentuates the need for higher levels and consistence application of MMT coverage.

However, because of the sensitivity of working on prisons in Iran, there is not much information available about the advantages and shortcomings of MMT in Iran. In a small scale study, Zamani et al. (34) carried out a research in only one prison in 2010 to determine the obstacles against the extension of MMT program from the viewpoints of prisoners which were lack of manpower and healthcare providers, methadone diversion, the potential adverse effects of the drug, and the stigma surrounding treatment by methadone. Recent studies in Iran have shown that activities of MMT centers have been cost-effective in HIV prevention and have averted about 128 new cases of HIV during only one year in 7 MMT sites (35) and also prevented HCV infection (36). Researchers in Malaysia recommended that prisonbased MMT program could be effectively implemented by improving a cogent link between prison and police authorities, preventing police harassment against MMT clients after their release, and improving systems for tracking release dates (28). Nowadays, there is now evidence-based information concerning the healthcare providers and mangers' opinion who are directly involved in the MMT program, although it has been proved that implementation of MMT along with other harm reduction strategies in recent years have considerably helped in reducing new HIV cases $(4,13)$. Having the feedback and opinions of those individuals who worked on and provided this program can be useful for health policy-makers in this context. Although MMT program has been widely used in prisons in Iran, to the best of our knowledge no study has been conducted yet to assess qualitatively the outcomes of integrating and running the program in prisons from the side of the program providers in Iran. Thus, in this qualitative study, we visited key people who were dealing with the implementation of MMT and we asked their perspective about MMT program in Iran's prisons. The aim of this study was to obtain the viewpoints of practitioners, consultants, experts, directors, and managers of prisons about the advantages and shortcomings of MMT programs in Iran's prisons.

\section{Methods}

Design and samples

This qualitative study was carried out in Iran's prisons. The main MMT providers including prison directors and managers, physicians and nurses, and consultants and psychologists of the prisons were enrolled in the study. The participants were recruited via purposive sampling with a heterogeneous approach. Those who had a history of less than six months of working with addicts, PWIDs, and people in need of MMT were excluded from the study. The people, participating in both types of Focus Group Discussions (FGDs), were from different prisons all around Iran. The researchers tried to select informant people in all prisons in Iran to participate in FGDs.

\section{Data collection}

Data were collected using FGDs. Since the participants were not in the same positions, we made the discussions separately for two different groups to form homogeneous group discussions (37). Thus, 14 separate FGDs (with 7 to 11 participants each) had been held, of which seven FGDs were carried out among prisons' directors and managers and seven FGDs were done among physicians, consultants and psychologists involved in the MMT program and worked at the triangular sites of the prisons participants were guided via an open discussion by a skilled facilitator/moderator. The moderator attempted to use the idea of all participants during the discussions was made confident every participated, asked for details if necessary and tried to make strong interactions 
among the subjects to reach to maximum and different opinions. These FGDs continued until the researchers found no fresh idea for each section. When the researchers realized that the discussions were not productive anymore, they decided to terminate or change the direction of the sessions to absorb the highest ideas. All FGDs were done at the health departments of the prisons. During FGDs, all the contents of participants' discussion were audio recorded and latter, after the discussion, their main ideas were copied in hand written notes. Participants were free to leave the study anytime they wished.

\section{Data analysis and validity}

The handwritten and audio recorded texts were analyzed via content analysis and thematic framework methods (38). On the basis of researchers' interpretation and deduction, after themes were extracted and encoded, similar codes were classified, integrated, and combined and the final results were extracted in accordance with the study objectives.

\section{Results}

Findings of this study included a total of 48 codes, some of which were related to advantages and some others to shortcomings of MMT program in prisons. Most participants in the study said that MMT program was very positive in prisons and they put emphasis on its constructive effects on harm reduction in prisons. They believed that despite some problems and weaknesses, MMT had been successful and had a good result on prisons and even on community. Generally, after coding contents and combining similar codes, the results were categorized as strengths and weaknesses which are listed separately below:

A) Participants' views about the efficacy of Methadone Maintenance Treatment (MMT) programs in prison (strengths)

Reduced shared injections

One of the most striking points concerning the MMT program in the view of the participants was its effects on reducing shared injections and subsequent reduction in the incidence of diseases which are transmitted through blood such as HIV and hepatitis.

\section{Reduced drug abuse in prison}

Participants said that the MMT program not only reduced the entry of drugs into the prison, but also decreased the need for, demand for, consumption, and trade of drugs and cigarettes.

\section{Reduced crime}

Participants believed that the MMT program could keep addicts calm in prison and could decrease crime; one of them said: "There has been an obvious reduction in escape, riot, tension, conflict and self-injury among the prisoners, especially addicts after the introduction of the MMT".

\section{Reduced consumption of drugs}

Officials and participants believed that MMT increased addicts' desire to quit drugs in prisons. They stated that: "After addicts are released from prison, they search for addiction treatment centers and counselors to continue their MMT programs".

\section{Reduced consumption of different types of drugs}

One of the officials said that: "Prior to the implementation of MMT in prisons, ecstasy pills were highly consumed even in front of prison officials. However, after the initiation of MMT, consumption of these pills decreased significantly".

\section{Positive economic, social, and behavioral effects}

Methadone brings addicts back to life. MMT program improves addicts' personal and social lives and brings them back to the community; it also creates a sense of responsibility and responsiveness in addicts who are under the treatment. This treatment also develops a positive attitude among family members and community toward addicts.

\section{Increased efficiency of training}

Participants believed that MMT made training and consulting programs more effective for addicts. One of the participants said: "Because of consumption of methadone, there is an increased level of enthusiasm to participate in sports and educational classes".

Table 1 presents some of the other most important advantages of MMT which were declared by doctors, consultants, experts, directors and managers of the prisons.

\section{B) Participants' views about shortcomings of Methadone} Maintenance Treatment (MMT) programs in prison (weakness)

Results of this study showed that although participants did not have a clear and strong disagreement with the implementation of this program, most of them believed that the program had not been implemented fully and was not in line with the designed protocols. After combining the codes, weaknesses and problems of this program were as follows (from participants' viewpoints):

\section{Lack of sufficient manpower}

Participants believed that the program was implemented incompletely due to shortage of staff; they also said that those who were working in prison were indeed the employees of other organizations. Accordingly, working in prison was not their first and main job. Thus, there was an unbalance between the number of employees and the number of prisoners who were under the coverage of the program.

\section{Inaccurate implementation of the protocol}

The program was not implemented along the lines of the protocol. In many cases, even the prison protocol was not fully implemented. "If this protocol is going to be implemented partially, we will obtain no positive feedback on its outcomes. Lack of proper, efficient and effective managerial strategies, especially in prisons is the main cause of the problem".

\section{Using methadone only as a relaxing tool in prison}

As one of the problems of the program, some of the normal and non-addicted prisoners voluntarily enrolled in the program in order to take methadone to pass detention period without any anxiety. Also, some less-addicted people chose methadone due to the sedation found in methadone. Using 
Table 1. Viewpoints of physicians, consultants, experts, directors and managers on the advantages (strengths) of methadone therapy

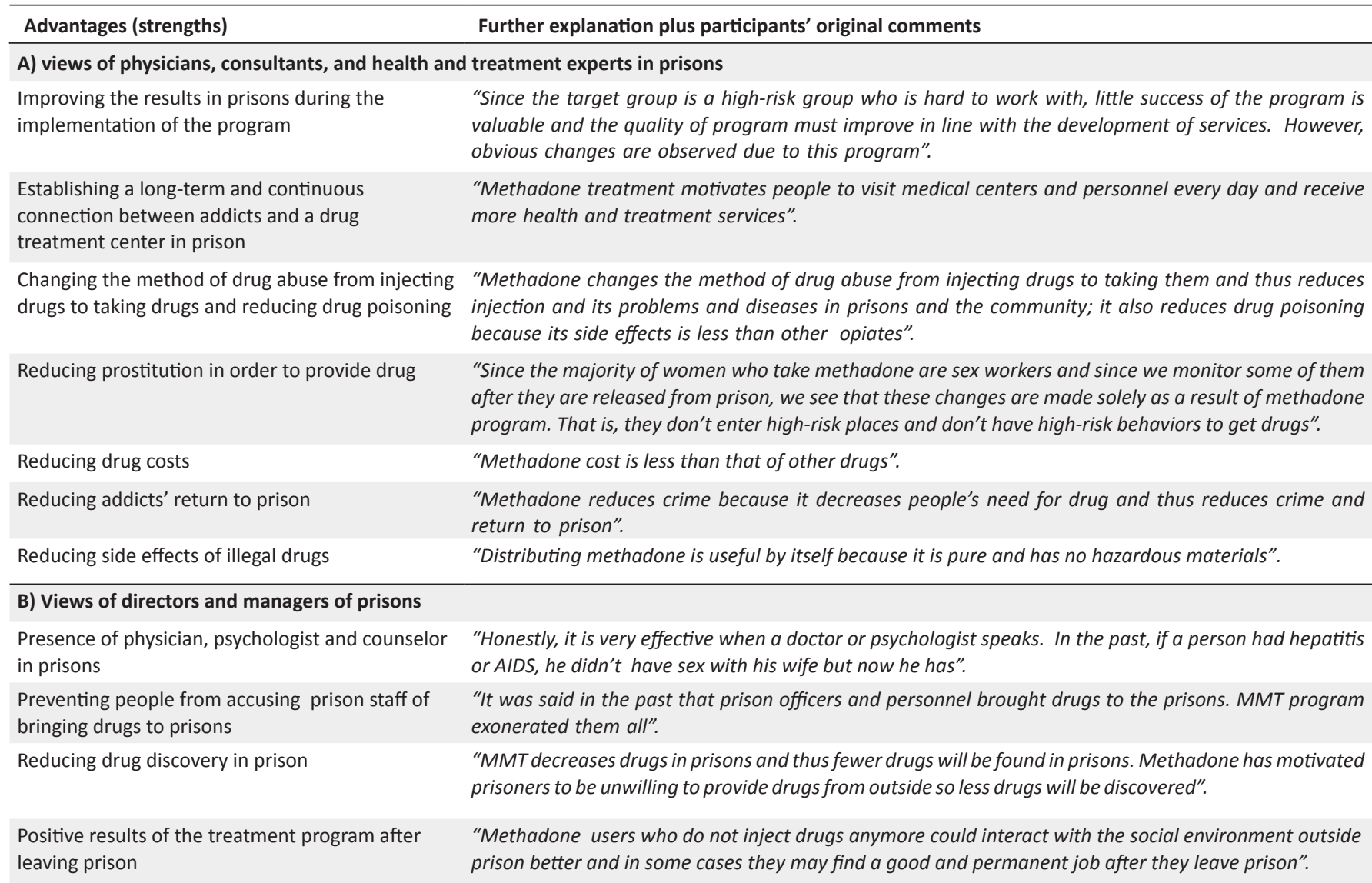

MMT= Methadone Maintenance Treatment.

the same treatment for all prisoners and ignoring differences between them has led to an increase in drug abuse. Methadone has short-term harm reduction effects, but in a long run it has many complications. "We give methadone to prisoners to calm them; it causes no problem for us and prevents them from fighting. It calms them here. But, what happens when they go back to community? We did not do what we have to do".

\section{Deficiencies after leaving prison}

Another major point which more than half of the participants pointed out was the problem of post-prison care. They believed that care provided outside the prisons was very weak and there was no special center to support and protect prisoners after their release. Moreover, inmates start drug abuse again after leaving prison. "If a person who enters the prison has the necessary indications, we include him in the treatment. The treatment mostly remains incomplete when he leaves the prison. Since treatment is free in prison, the prisoner wants to find free centers out of the prison but unfortunately there is no such a center outside".

Low awareness of the staff towards the program

Participants reported that both drug-dependant users and staff who provided services and executed the program are not much aware of the principal objectives of the MMT program. They also stated that staff are not experienced; and personnel are not fully familiar with the prison environment.

Lack of proper criteria for inclusion of people in the program The program is not implemented scientifically since all addicts and even non-addicts are included in the methadone program. "As another problem of methadone plan, at first the authorities commended, in keeping with the first protocol, to only include people who had high-risk behaviors like those who injected drugs and those who used heroine or crack. But then, they told us to include everyone who wanted to use methadone". Other shortcomings mentioned by physicians, consultants, experts, directors and managers of prisons are summarized in Table 2.

\section{Discussion}
In this study, we identified positive points of the MMT program such as reduced injection, reduced demand and subsequent reduction in the availability and abuse of drugs in prison, increased tendency to withdraw, decreased consumption of narcotics and sedatives, emergence of a positive attitude in families and community, increased efficiency of training and consulting classes, establishing long-term and continuous connections between addicts and treatment centers or counseling teams, reduced rate of crimes like prostitution in order to obtain drugs, and reduced addiction expenses. on the other side, participants presented challenges of this program including inaccurate implementation of the designed protocols, shortage of skilled manpower and service providers, weakness in post-release continuity of care, low awareness of groups and community about various aspects of the program, lack of proper and efficient criteria for including people in the program, neglecting psychological problems of addicts, wrong policies 
Table 2. Viewpoints of physicians, consultants, experts, directors and managers on the weaknesses of the program

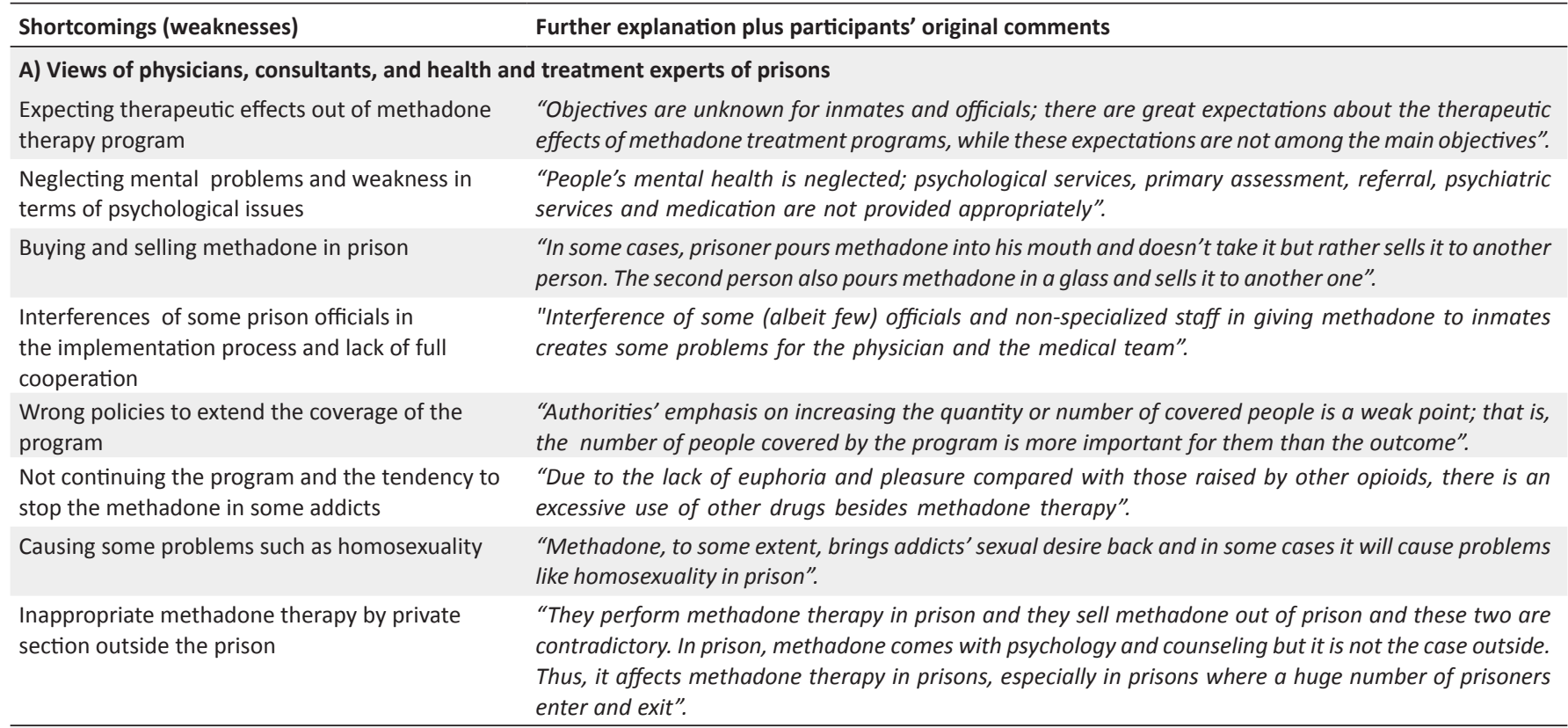

\section{B) Views of directors and managers of prisons}

Lack of proper and valid indicators to assess the progress and success of the program

Shortage of space and facilities necessary to implement the program completely and appropriately

Entering the program due to financial problems (prisoners' poverty)

Unfamiliarity of judges with the program

Stopping methadone as a punishment

Paying more attention to big prisons and having problems in implementing the program in small prisons

Making threats by prisoners to receive methadone
"Now, there's no appropriate measure to evaluate the progress of the program clearly and definitely".

"Shortage of budget, staff, equipment and facilities of centers. These deficiencies sometimes cause the programs to be stopped".

"In some cases, people select methadone because of poverty, not because of withdrawal".

"The judiciary does not completely agree with implementing such programs because judges are not aware of them and this causes problems in the programs".

In some prisons, officials make the policy of stopping methadone as a punishment. They say: "Don't give them the methadone".

"The focus on improving the program can be only seen in big prisons. In smaller prisons, however, methadone programs are not implemented as properly as the big ones; its implementation in small prisons is hard because of the shortage of facilities".

"Prisoners threaten personnel to receive drugs and methadone and even commit intentional crimes to receive methadone. Sometimes, prisoners, themselves, select dose of methadone." in increasing the coverage of methadone therapy, slow implementation, and shortage of valid evaluation criteria. There is growing scientific evidence that indicates commencing MMT before releasing from prison declines recurrent post-release substance use. McKenzie et al. (39) said that despite notable provider-related barriers to implement MMT for drug users in related centers and correctional settings, it is necessary to provide effective intervention methods via supplying methadone before releasing the prisoners. Nonetheless, one of the important and controversial points about MMT programs in Iran prisons is that other organizations do not support this program at social level and do not provide services for released prisoners. Clearly, some cities still lack methadone centers or centers for post-prison care; furthermore, other governmental centers do not accept addicts who are released from prisons. Moreover, since these people are poor, they are not able to find a job so they are likely to get addicted again after they are released from prison. It can be concluded that harm reduction and addiction treatment would become incomplete without the cooperation of other organizations outside the prison. A study, carried out in Belgium by De Maeyer et al. (15), emphasized the role of social and environmental support for addicts treated with methadone in order to increase quality of their lives. Another study conducted in Yunnan province of China indicated that some factors can influence the success of the program, including increased awareness of community and drug users about methadone, behavior therapy along with counseling, vocational training, increased interaction with the police and judicial authorities and the relationship with public health system (40).

Generally speaking, the objectives of methadone therapy in prisons are still not clear and obviously MMT is introduced as a way to reduce harm; if therapeutic effects is the main objective, some cases must be taken into consideration including presenting protocols with certain objectives and implementing the programs exactly. In other words, to understand methadone therapy completely, common objectives of withdrawal programs must be also taken into account: 1) Eliminating or reducing withdrawal symptoms, 2) Eliminating or reducing the craving for drugs, 3) Preventing recurrence of addiction, 4) Restoring the physiological activity level which has been lost due to drug abuse, and 5) Reducing criminal behaviors and improving behaviors that affect social 
and psychological health $(27,41-43)$.

Iran is considered as one of the most successful countries in the field of methadone therapy as a method of harm reduction (44), however, various studies conducted in other countries have also shown the success of methadone therapy in harm reduction, especially in reducing high-risk behaviors such as shared injection $(15,19,28,41,45,46)$. Thus, according to the results of the present research, the weaknesses extracted from these programs can be a guide to review and continue MMT programs in Iran prisons. When its weaknesses are removed via scientific methods and its different aspects are supported by other non-governmental and governmental organizations, MMT can potentially reduce harm and lead to good therapeutic effects. There should be a strategic plan for this program in prisons. Such a plan should define clear standards for exclusion and inclusion of eligible individuals. The program should also undergo continuous monitoring and evaluation.

There are some limitations for the current study. One of the main drawbacks of this study is that authors would like to ask the perspectives of the receivers of MMT programs, although other studies like Zamani et al. (34) explored the main positive and negative points of the MMT program from the prisoners' view. Another one is that this study was a qualitative approach to explore the weakness and strength of MMT program; however, qualitative studies do not have rigorous power to show the main factors or elements of a program such as MMT; therefore, there is a need to make these qualitative themes to quantitative factors and explore the main positive and negative factors in figures and statistics.

\section{Conclusion}

According to the findings of this research, it can be concluded that MMT programs have had considerable success not only in reducing drug-related harms, but also in improving prisoners' lifestyle. However, to achieve more success it is necessary to recruit skilled manpower in all prisons, provide specific guidelines with certain objectives, implement the guidelines properly, and mobilize various governmental and non-governmental organizations. Because of its potential effect on harm reduction activities, it can reduce the risky behaviors like shared injection and the use of different illegal drugs, and lead to shifts from injection to non-injection use of drugs. Moreover, it can prevent the transmission of infectious diseases like HIV among prisoners. Providing sustainable and long-term cares, even after release form the prisons, is another option which has to be considered by health and administrative authorities.

\section{Acknowledgments}

The authors would like to express sincere thank to Iran Prisons staff for their cooperation in FGDs and sharing their knowledge and experience, and also we would like to thank Prisons Organization for their coordination to arrange the regional meetings.

\section{Ethical issues}

To meet the ethical issues in research, participants' voices were recorded only after obtaining their consent; in case of participants' reluctance, their voices were not recorded and just notes were taken. The participants were also assured that the content of their discussions would remain confidential and that their identities would by no means be disclosed. Informed verbal consent was obtained from all participants in each FGD.

\section{Competing interests}

The authors declare that they do not have any conflict of interests for this article. However, we would like to mention that the author "Marzieh Farnia" is the member of Iran Prisons Organization.

\section{Authors' contributions}

Concept of the study: GM, MF, and MSha; study design: GM and MSha; collecting the data: MSha and KR; Analyzing the data: MSho, BM, MSha and KR; interpretation of the results: MSho, MSha, GM, BM and KR; drafting the manuscript: MSha, MSho, GM, KR and BM. All authors have read and approved the content and the authorship of the final version of the submitted manuscript.

\section{Authors' affiliations}

${ }^{1}$ Kurdistan Research Center for Social Determinants of Health (KRCSDH), Kurdistan University of Medical Sciences, Sanandaj, Iran. ${ }^{2}$ Iran Prisons Organization, Health and Treatment Department, Tehran, Iran. ${ }^{3}$ Regional Knowledge Hub, and WHO Collaborating Centre for HIV Surveillance, Institute for Futures Studies in Health, Kerman University of Medical Sciences, Kerman, Iran. ${ }^{4}$ GFATM Projects in Prisons, United Nations Development Program, Tehran, Iran. ${ }^{5}$ Non-Communicable Diseases Research Center, Endocrinology and Metabolism Population Sciences Institute, Tehran University of Medical Sciences, Tehran, Iran. ${ }^{6}$ Endocrinology and Metabolism Research Center, Endocrinology and Metabolism Clinical Sciences Institute, Tehran University of Medical Sciences, Tehran, Iran. ${ }^{7}$ School of Public Health, Shahid Beheshti University of Medical Sciences, Tehran, Iran.

\section{References}

1. Navadeh S, Mirzazadeh A, Gouya MM, Farnia M, Alasvand $R$, Haghdoost AA. HIV prevalence and related risk behaviours among prisoners in Iran: results of the national biobehavioural survey, 2009. Sex Transm Infect 2013; 89: iii33-6. doi: 10.1136/ sextrans-2013-051295

2. Zamani S, Kihara M, Gouya MM, Vazirian M, Nassirimanesh B Ono-Kihara M, et al. High prevalence of HIV infection associated with incarceration among community-based injecting drug users in Tehran, Iran. J Acquir Immune Defic Syndr 2006; 42: 342-6. doi: 10.1097/01.qai.0000219785.81163.67

3. Stahl W, Sies H. beta-Carotene and other carotenoids in protection from sunlight. Am J Clin Nutr 2012; 96: 1179S-84S. doi: $\quad$ 10.3945/ajcn.112.034819

4. Haghdoost AA, Mirzazadeh A, Shokoohi M, Sedaghat A, Gouya MM. HIV trend among Iranian prisoners in 1990s and 2000s; analysis of aggregated data from HIV sentinel sero-surveys. Harm Reduct J 2013; 10: 32. doi: 10.1186/1477-7517-10-32

5. Beyrer C, Jittiwutikarn J, Teokul W, Razak MH, Suriyanon V, Srirak $\mathrm{N}$, et al. Drug use, increasing incarceration rates, and prison-associated HIV risks in Thailand. AIDS Behav 2003; 7 : 153-61.

6. Macalino GE, Vlahov D, Sanford-Colby S, Patel S, Sabin K, Salas C, et al. Prevalence and incidence of HIV, hepatitis B virus, and hepatitis $\mathrm{C}$ virus infections among males in Rhode Island prisons. Am J Public Health 2004; 94: 1218-23.

7. Vanichseni S, Kitayaporn D, Mastro TD, Mock PA, Raktham $\mathrm{S}$, Des Jarlais DC, et al. Continued high HIV-1 incidence in a vaccine trial preparatory cohort of injection drug users in Bangkok, Thailand. AIDS 2001; 15: 397-405.

8. Kushwaha S, Kramer J, Kates M. Isolation and characterization of C50-carotenoid pigments and other polar isoprenoids from Halobacterium cutirubrum. Biochimica et Biophysica Acta (BBA)Lipids and Lipid Metabolism 1975; 398: 303-14.

9. Dolan KA, Wodak A. HIV transmission in a prison system in an Australian State. Med J Aust 1999; 171: 14-7.

10. Taylor A, Goldberg D, Emslie J, Wrench J, Gruer L, Cameron S, et al. Outbreak of HIV infection in a Scottish prison. BMJ 1995; 310: 289-92.

11. Razzaghi E, Nassirimanesh B, Afshar P, Ohiri K, Claeson M, 
Power R. HIVIAIDS harm reduction in Iran. Lancet 2006; 368: 434-5. doi: 10.1016/s0140-6736(06)69132-0

12. Dolan KA, Shearer J, MacDonald M, Mattick RP, Hall W, Wodak $\mathrm{AD}$. A randomised controlled trial of methadone maintenance treatment versus wait list control in an Australian prison system. Drug Alcohol Depend 2003; 72: 59-65.

13. Farrel M, Gowing L, Marsden J, Ling W, Ali R. Effectiveness of drug dependence treatment in HIV prevention. International Journal of Drug Policy 2005; 16S: 67-75.

14. Gossop M, Marsden J, Stewart D, Treacy S. Outcomes after methadone maintenance and methadone reduction treatments: Two-year follow-up results from the National Treatment Outcome Research Study. Drug Alcohol Depend 2001; 62: 255-64.

15. De Maeyer J, Vanderplasschen W, Camfield L, Vanheule S, Sabbe B, Broekaert E. A good quality of life under the influence of methadone: a qualitative study among opiate-dependent individuals. Int J Nurs Stud 2011; 48: 1244-57. doi: 10.1016/j. ijnurstu.2011.03.009

16. De Maeyer J, Vanderplasschen W, Lammertyn J, van Nieuwenhuizen C, Sabbe B, Broekaert E. Current quality of life and its determinants among opiate-dependent individuals five years after starting methadone treatment. Qual Life Res 2011; 20: 139-50. doi: 10.1007/s11136-010-9732-3

17. Heimer R, Catania H, Newman RG, Zambrano J, Brunet A, Ortiz AM. Methadone maintenance in prison: evaluation of a pilot program in Puerto Rico. Drug Alcohol Depend 2006; 83: 122-9. doi: 10.1016/j.drugalcdep.2005.11.004

18. Kinlock TW, Gordon MS, Schwartz RP, Fitzgerald TT, O'Grady $\mathrm{KE}$. A randomized clinical trial of methadone maintenance for prisoners: results at 12 months postrelease. $J$ Subst Abuse Treat 2009; 37: 277-85. doi: 10.1016/j.jsat.2009.03.002

19. Larney S, Toson B, Burns L, Dolan K. Effect of prison-based opioid substitution treatment and post-release retention in treatment on risk of re-incarceration. Addiction 2012; 107: 37280. doi: 10.1111/j.1360-0443.2011.03618.x

20. Ball JC, Lange WR, Myers CP, Friedman SR. Reducing the risk of AIDS through methadone maintenance treatment. Journal of Health and Social Behavior 1988; 29: 214-26.

21. Morrow K, Costello T. HIV, STD and hepatitis prevention among women in methadone maintenance: a qualitative and quantitative needs assessment. AIDS Care 2004; 16: 426-33. doi: $\quad 10.1080 / 09540120410001683367$

22. Langendam MW, van Brussel GH, Coutinho RA, al. e. The impact of harm-reduction-based methadone treatment on mortality among heroin users. Am J Public Health 2001; 91: 774-80.

23. Mattick RP, Breen C, Kimber J, Davoli M. Methadone maintenance therapy versus no opioid replacement therapy for opioid dependence. Cochrane Database Syst Rev 2009; (3): CD002209

24. Cabral M, Cence K, Zeni J, Tsai S, Durrer A, Foltran L, et al. Carotenoids production from a newly isolated Sporidiobolus pararoseus strain by submerged fermentation. Eur Food Res Technol 2011; 233: 159-66. doi: 10.1007/s00217-011-1510-0

25. Dolan KA, Shearer J, White B, Zhou J, Kaldor J, Wodak AD. Fouryear follow-up of imprisoned male heroin users and methadone treatment: mortality, re-incarceration and hepatitis $\mathrm{C}$ infection. Addiction 2005; 100: 820-8.

26. Acosta MC, Marsch LA, Xie H, Guarino H, Aponte-Melendez Y. A web-based behavior therapy program influences the association between cognitive functioning and retention and abstinence in clients receiving methadone maintenance treatment. J Dual Diagn 2012; 8: 283-93. doi: 10.1080/15504263.2012.723317

27. Lin C, Wu Z, Rou K, Pang L, Cao X, Shoptaw S, et al. Challenges in providing services in methadone maintenance therapy clinics in China: service providers' perceptions. Int J Drug Policy 2010; 21: 173-8. doi: 10.1016/j.drugpo.2009.09.002

28. Wickersham JA, Marcus R, Kamarulzaman A, Zahari MM, Altice $\mathrm{FL}$. Implementing methadone maintenance treatment in prisons in Malaysia. Bull World Health Organ 2013; 91: 124-9. doi: 10.2471/blt.12.109132
29. Wickersham JA, Zahari MM, Azar MM, Kamarulzaman A, Altice FL. Methadone dose at the time of release from prison significantly influences retention in treatment: implications from a pilot study of HIV-infected prisoners transitioning to the community in Malaysia. Drug Alcohol Depend 2013; 132: 37882. doi: 10.1016/j.drugalcdep.2013.01.005

30. Sheerin I, Green T, Sellman D, Adamson S, Deering D. Reduction in crime by drug users on a methadone maintenance therapy programme in New Zealand. N Z Med J 2004; 117: U795.

31. Eshrati B, Taghizadeh-AsI R, Dell CA, Afshar P, Millson PME, Kamali M, et al. Preventing HIV transmission among Iranian prisoners: Initial support for providing education on the benefits of harm reduction practices. Harm Reduct J 2008; 5: 21.

32. Larney S, Dolan K. A literature review of international implementation of opioid substitution treatment in prisons: Equivalence of care? Eur Addict Res 2009; 15: 107-12.

33. Manikandan $\mathrm{M}$, Hasan N, Wu H-F. Rapid detection of haloarchaeal carotenoids via liquid-liquid microextraction enabled direct TLC MALDI-MS. Talanta 2013; 107: 167-75.

34. Zamani S, Farnia M, Tavakoli S, Gholizadeh M, Nazari M, Seddighi $A A$, et al. A qualitative inquiry into methadone maintenance treatment for opioid-dependent prisoners in Tehran, Iran. Int J Drug Policy 2010; 21: 167-72. doi: 10.1016/j. drugpo.2009.03.001

35. Keshtkaran A, Mirahmadizadeh AR, Heidari AR, Javanbakht $M$. Cost-effectiveness of methadone maintenance treatment in prevention of hiv among drug users in Shiraz, south of Iran. Iran Red Cres Med J 2014; 16: e7801.

36. Alavian SM, Mirahmadizadeh A, Javanbakht M, Keshtkaran A, Heidari A, Mashayekhi A, et al. Effectiveness of methadone maintenance treatment in prevention of hepatitis $\mathrm{C}$ virus transmission among injecting drug users. Hepat Mon 2013; 13: e12411. doi: 10.5812/hepatmon.12411

37. Krueger RA. Focus groups: a practical guide for applied research. 2nd ed. Thousand Oaks, CA: Sage Publications; 1994.

38. Ritchie J, Spencer L, O'Connor W. Carrying out qualitative analysis. In: Ritchie J, Lewis J, editors. Qualitative research practice: A guide for social science students and researchers. London: Sage Publications; 2004. p. 219-62.

39. McKenzie M, Nunn A, Zaller ND, Bazazi AR, Rich JD. Overcoming obstacles to implementing methadone maintenance therapy for prisoners: implications for policy and practice. J Opioid Manag 2009; 5: 219-27.

40. Philbin MM, Zhang F. Exploring stakeholder perceptions of facilitators and barriers to accessing methadone maintenance clinics in Yunnan Province, China. AIDS Care 2010; 22: 623-9. doi: 10.1080/09540120903311490

41. Hedrich D, Alves P, Farrell M, Stover H, Moller L, Mayet S. The effectiveness of opioid maintenance treatment in prison settings: a systematic review. Addiction 2012; 107: 501-17. doi: 10.1111/j.1360-0443.2011.03676.x

42. Lin C, Detels R. A qualitative study exploring the reason for low dosage of methadone prescribed in the MMT clinics in China. Drug Alcohol Depend 2011; 117: 45-9. doi: 10.1016/j. drugalcdep.2011.01.004

43. Lin C, Wu Z, Detels R. Opiate users' perceived barriers against attending methadone maintenance therapy: a qualitative study in China. Subst Use Misuse 2011; 46: 1190-8. doi: 10.3109/10826084.2011.561905

44. Farnia M, Ebrahimi B, Shams A, Zamani S. Scaling up methadone maintenance treatment for opioid-dependent prisoners in Iran. Int J Drug Policy 2010; 21: 422-4. doi: 10.1016/j. drugpo.2010.03.008

45. Magura S, Rosenblum A, Lewis C, Joseph H. The effectiveness of in-jail methadone maintenance. J Drug Issues 1993; 23: 75.

46. Smye V, Browne AJ, Varcoe C, Josewski V. Harm reduction, methadone maintenance treatment and the root causes of health and social inequities: An intersectional lens in the Canadian context. Harm Reduct J 2011; 8: 17. doi: 10.1186/1477-7517$8-17$ 